\title{
Evaluating the use of computerised clinical guidelines in the accident and emergency department
}

\author{
H D M Poncia, G D R Bryant, J Ryan
}

\begin{abstract}
Objectives-To investigate the pattern and frequency of use of computerised clinical guidelines (CCG) in an accident and emergency department.
\end{abstract}

Methods-A software program was written to record information on a central database each time the CCG were used. Data were collected prospectively for a six month period. Users were blind to the study. The date, time of use and guidelines consulted were recorded.

Results-1974 individual sessions were logged comprising of 10204 "hits". The CCG were used for a median of 10 sessions per day (range 1-38, SD 5.49). A median of three subjects were accessed during each session (range 1-39, SD 5). The CCG were used most often during peak daily activity; 11 am (609 hits), 5 pm (678 hits) and 12 pm (604 hits) and on Sundays (1875 hits), Thursdays (1770 hits) and Saturdays (1608 hits). The most frequently used guidelines concerned orthopaedics and fracture management (1590 hits), wound care (546 hits), poisoning (473 hits), medical emergencies (267 hits) and radiological policy (148 hits).

Conclusions-In this department CCG have become easily integrated as part of normal day to day working practice. The CCG are accessible 24 hours a day. They can also be easily updated according to best evidence, local policy or national guidelines. The results of this study have helped the authors to focus education to areas of clinical need.

(F Accid Emerg Med 2000;17:254-256)

Keywords: guidelines; information; computer

As the philosophy of multi-disciplinary care, integrated care pathways and evidence based practice extends within the accident and emergency (A\&E) department, it is increasingly important that all $\mathrm{A} \& \mathrm{E}$ staff have easy access to agreed departmental guidelines and protocols. In our department we have developed the use of computerised clinical guidelines (CCG). These are accessible from any of 14 computer terminals located throughout the department and because of this, information is easily accessible to medical or nursing staff in a matter of seconds, 24 hours a day. The CCG consist of 517 "pages" in Windows Help file format covering 502 subjects. There is extensive cross referencing with hyperlinks between related subjects.

The objectives of this study were to evaluate the pattern and frequency of use of CCG in the A\&E department.

\section{Methods}

The CCG software was modified so that each time the guidelines were accessed a record was logged on a central database. Users were unaware of the study. Data were collected prospectively for a six month period. A "session" consisted of more than one page visited in
Department of Accident and

Emergency Medicine, Royal Sussex County Hospital, Eastern Road, Brighton BN2 5BE

Correspondence to: Mr Ryan

(ryanj@pavilion.co.uk)

Accepted for publication 23 October 1999

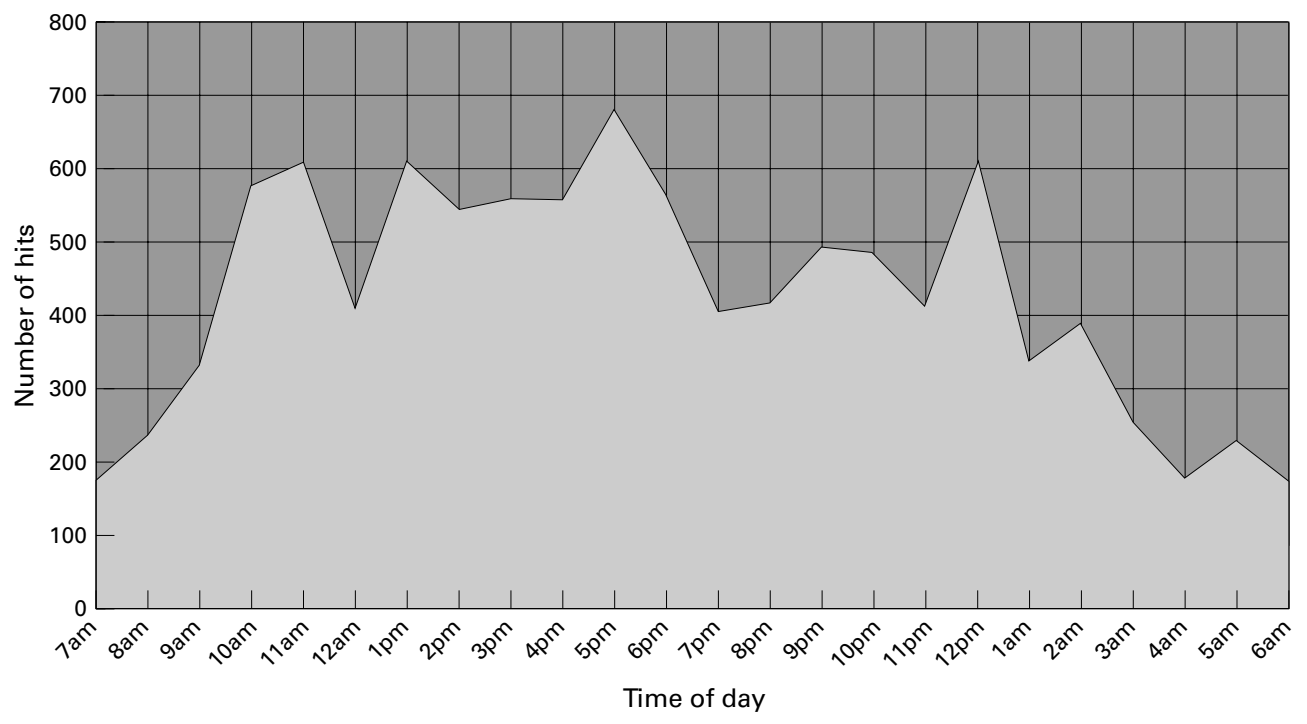

Figure 1 Distribution of total hits according to hour of the day. 


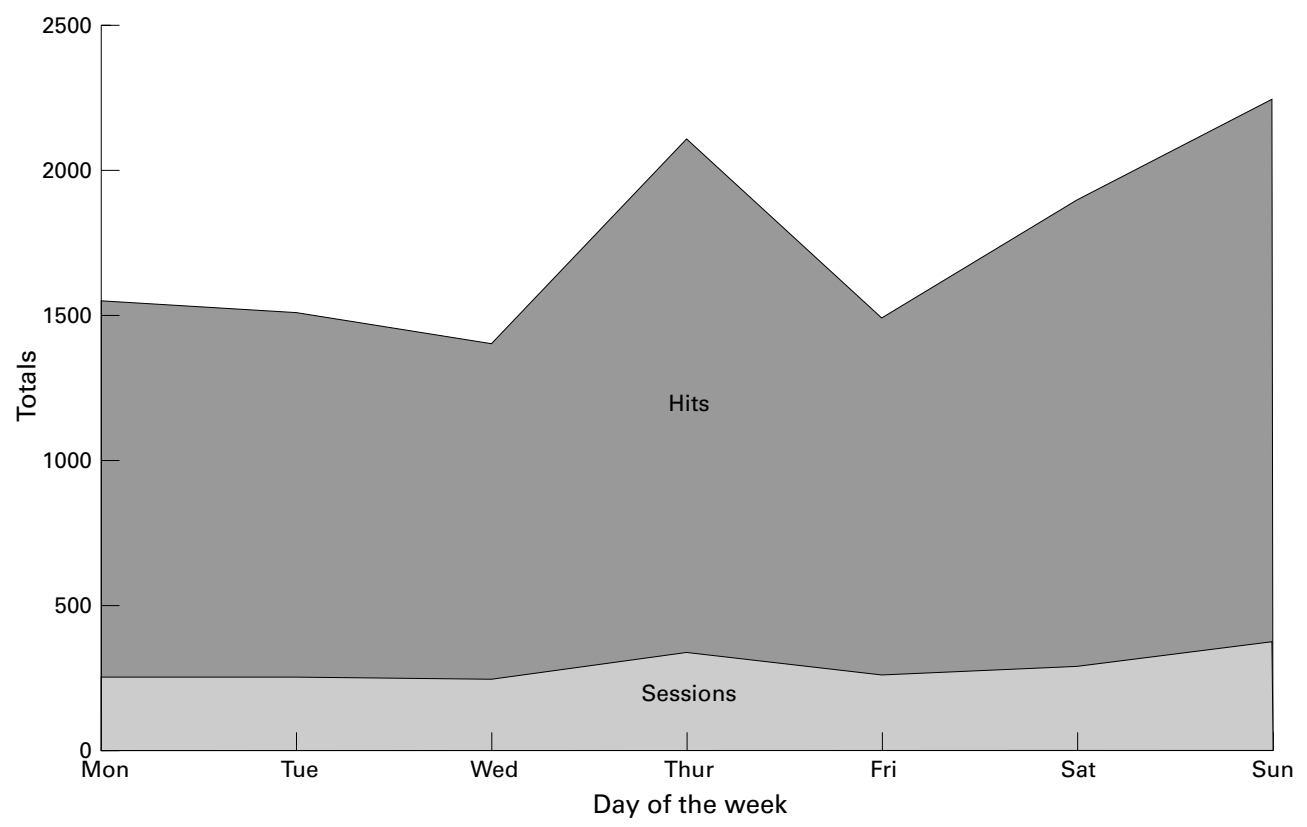

Figure 2 Total number of sessions and hits per day of the week.

sequence at the same location at the same time. A "hit" was recorded for each "page" visited. The date, time of access and subjects consulted were recorded for every session.

\section{Results}

A total of 1974 individual sessions were logged, comprising of 10204 hits. The CCG were used for 10 sessions per day (median 10, range 1-38, SD 5.49). A median of three subjects were accessed during each session (range 1-39, SD 5). CCG were used most often during periods of peak daily activity; 11 am (609 hits),

\section{Subjects}

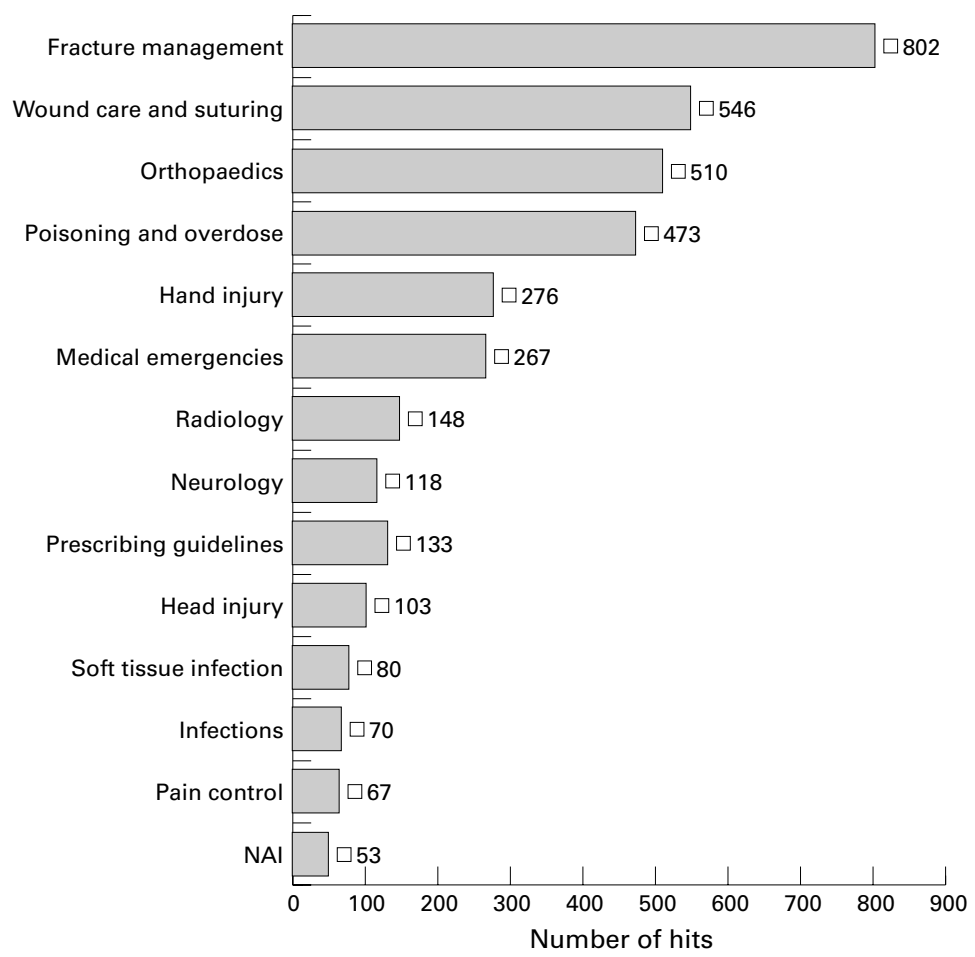

Figure 3 Top 14 subjects accessed by users.
5 pm (678 hits) and 12 pm (604 hits) (fig 1). Sundays (1875 hits), Thursdays (1770 hits) and Saturdays (1608 hits) were the busiest days for CCG use (fig 2). The top five subjects accessed were orthopaedics and fracture management (1590 hits), wound care (546 hits), poisoning (473 hits), medical emergencies (267 hits) and radiological policy (148 hits) (fig 3).

\section{Discussion}

Clinical guidelines are becoming an increasingly familiar part of clinical practice. They can improve the quality of clinical decision making by promoting interventions of proved benefit and discouraging ineffective ones. They may improve efficiency and reduce outlays for hospitilisation, surgery, prescription drugs and other procedures. They can also improve consistency of care and have the potential to reduce morbidity and mortality. ${ }^{1}$ However, if effective guidelines are to be followed in the $\mathrm{A} \& \mathrm{E}$ department, then they must be easily accessible day or night by every member of a multiprofessional workforce. Fortunately the development of information technology systems and the development of CCG have made this easily achievable.

\section{ADVANTAGES OF CCG}

Emergency health care workers are among the most information intensive of healthcare professionals. ${ }^{2}$ The breadth of clinical problems encountered in the $\mathrm{A} \& \mathrm{E}$ department is large and time can be a precious commodity. Historically in the United Kingdom access to clinical information by medical or nursing staff within busy A\&E departments has been restricted to paper based systems such as handbooks, folders, pamphlets or departmental textbooks. Quick and easy access to relevant and accurate clinical information can be a difficult process. Libraries are often locked, handbooks can be limited by their size, expensive to 
publish, rapidly go out of date and are easily lost. CCG can provide an effective solution for many of these problems by being quick and easy to use and readily available in every work area 24 hours a day. The pace of change in emergency medicine mandates frequent updates of departmental guidelines and protocols. CCG can be quickly and easily updated according to best evidence, local policy or national guidelines with minimal additional expenditure. The software format of these guidelines facilitates easy sharing with other units. This could facilitate consistent treatment by $A \& E$ departments regionally and nationally. The use of CCG may decrease the demand on limited specialist advisory units such as The National Poisons Information Unit for advice and consequently less use of the telephone.

EASE OF ACCESS

CCG are potentially much quicker to access than paper based systems, but there is an important trade off; systems in which it is easy to enter data tend to have slower rates of data retrieval. Systems with rapid rates of data retrieval tend to have slower and more complex methods of data entry. Rapid data retrieval is the more important. Systems with slow retrieval rates easily become neglected. There are a variety of different formats available however, Windows helpfiles have one of the most rapid rates of data retrieval available. It is our experience that CCG in this format are an effective tool.

PATTERN OF USE

The use of CCG closely mirrored periods of peak activity (figs 1 and 2). CCG can be used quickly and easily even when the department is very busy. They save time and thus enable $A \& E$ doctors and nursing staff to work more efficiently. In our study CCG were also used more often when no senior A\&E doctor was available. While we do not propose that CCG are in any way a substitute for supervision, they do function as a valuable adjunct.

\section{SUBJECTS ACCESSED}

The most frequently visited pages related to the management of orthopaedic problems, wound care/suturing and poisoning/overdose (fig 3). This reflects the high incidence of these problems within our patient case mix, but also that these are common areas of difficulty for $A \& E$ staff. A\&E doctors may feel unprepared for work within A\&E departments because comparatively little emphasis is placed upon some of these areas in the undergraduate curriculum. We have incorporated a computer based Service Based Learning package into the CCG, and as a result of our findings we have modified our local and regional induction programs for $\mathrm{A} \& \mathrm{E}$ doctors to reflect these areas of educational need.

RESEARCH

Information technology has much to offer A\&E departments, in addition to its existing role as an audit tool it can be an invaluable source of information. Information needs for clinical emergency medicine practice and emergency medicine research are also closely related. ${ }^{3}$ This paper shows that a well developed information system can serve both, allowing data gathered in one setting to be used for the other.

\section{Conclusions}

In our department CCG have become easily integrated as part of normal day to day working practice. They can be accessed 24 hours a day and are easily updated according to best evidence, local policy or national guidelines. This technology is rapidly evolving and its full potential has yet to be realised. The practice of medicine is dominated by how we process, record, retrieve and communicate information. ${ }^{4}$ The emergency department of the future will require the effective integration of information technologies into clinical care. ${ }^{2}$ We recommend that CCG be developed further for wider use in A\&E departments.

Contributors

Hugo Poncia analysed the data and wrote the paper. Geoff Bryant developed the guidelines, wrote the programme to record the data and edited the paper. John Ryan assisted writing and editing the paper. Mr Bryant and Mr Ryan are guarantors for the paper. Parties interested in developing the guidelines for use in their own departments should contact Mr Bryant at The Department of Accident and Emergency Medicine, Royal Sussex County Hospital, Eastern Rd, Brighton, BN2 5BE (bryantg@pavilion.co.uk).

Funding: none.

Conflicts of interest: none.

1 Woolf SH, Grol R, Hutchinson A, et al. Clinical guidelines; potential benefits, limitations and harms of clinical potential benefits, limitations and

2 Cordell WH, Overhage JM, Waeckerle JF. Strategies for improving information management in emergency medicine to meet clinical, research, and administrative needs. Ann Emerg Med 1998;31:172-8.

3 Teich JM. Information systems support for emergency medicine. Ann Emerg Med 1998;31:304-7.

4 Barnett O. Computers in medicine. $尹 A M A$ 1990;263:26313. 